\title{
List of Charts and Tables
}

Chart 1: Mentions of the "captive nations" and "Captive Nations Week" (CNW) in the Congressional Record, 1956-1989 190

Chart 2: Representatives and Senators mentioning the ACEN in U.S. Congress by state, 1955$1965-193$

Chart 3: Budget for ACEN Activities in Latin America 1962/63-1963/64 268

Tab. 1: ACEN Leadership, 1954-1972 - 31

Tab. 2: Top personnel for ACEN suggested by Bernard Yarrow, $1960-66$

Tab. 3: Latin American Tour by ACEN Delegates, 17 IX -18 X $1956-248$

Tab. 4: ACEN's Latin American delegations, 1956-1957 (Third Session of the ACEN) — 256

Tab. 5: ACEN Latin American delegations, 1962-1963 - 267

Tab. 6: ACEN-coordinated broadcasts in Latin America, $1964-277$

\section{Appendix:}

Tab. 7: ACEN Delegates by National Council/Committee, 1954-1972 - $359 \mathrm{ff}$.

Tab. 7A: Albania — 359

Tab. 7B: Bulgaria — 364

Tab. 7C: Czechoslovakia - 367

Tab. 7D: Estonia 372

Tab. 7E: Hungary -377

Tab. 7F: Latvia 383

Tab. 7G: Lithuania - 388

Tab. 7H: Poland - 391

Tab. 7l: Romania - 398

Tab. 8: Party internationals' delegations to ACEN, 1954-1971 - $401 \mathrm{ff}$.

Tab. 8A: CDUCE - 401

Tab. 8B: IPU 403

Tab. 8C: LDUCEE — 406

Tab. 8D: SUCEE — 408

Tab. 8E: ICFTUE - 409

Tab. 9: ACEN representations abroad, 1956-1971 $-411 \mathrm{ff}$. 
